\title{
Band gap engineering of hybrid 2-D nanomaterials some recent developments
}

\begin{abstract}
Successful modifications of band gaps of bulk alloy semiconductors (i.e. in binary, ternary and quaternary compounds) for electronic and optoelectronic device applications encouraged the researchers to explore similar strategies in 2D-materials involving graphene and graphene like transition metal chalcogenides and other layered materials. Being atomically thin layers, there are numerous other possibilities to explore in these 2D-materials involving lateral, vertical heterostructure formations besides substitution, and doping of other atomic species to fix their band gaps somewhere between zero of the graphene and the wide band gap, for example, of h-BN (i.e. $6 \mathrm{eV}$ band gap) in hybrid type h-BNC lattice. The recent developments taking place in this important area of research in band gap engineering of 2D-hybrid materials is briefly discussed in this review.
\end{abstract}

Volume I Issue 2 - 2017

\author{
Ahmad S \\ YMCA University of Science \& Technology, India \\ Correspondence: Ahmad S, YMCA University of Science \& \\ Technology, Faridabad, Haryana, India, \\ Email drsahmad@email.com
}

Received: April 30, 2017| Published: May 31, 2017

Keywords: optical transition, stoichiometric, superlattices, molecular beam, nanoscience, graphene, degradation, heterostructures, biomolecular, quantum, targeted therapy

Abbreviations: CCSM, crystalline condensed state material; EEBS, electron energy band structure; BGE, band-gap engineering; NPMs, nanoparticulate materials; NCs, nanocrystals; DES, discrete electron states; GBs, grain boundaries; SB, schottky barrier

\section{Introduction}

Physico-chemical properties of a crystalline condensed state material (CCSM) are decided by its electron energy band structure (EEBS) generally computed theoretically and validated by optical transition studies. Modifying the EEBS of such a CCSM would certainly amount to engineering its properties. Consequently, a number of studies were carried out in the context of bandgap engineering (BGE) of a large number of semiconducting materials by either changing their stoichiometric compositions in thermodynamically stable alloys or constructing layered epitaxial thin films with alternating compositions with matching lattice constants at the interfaces involving appropriate elemental and compound semiconductors. BG controls could facilitate easy access to their modified electronic/optoelectronic properties usable in respective device applications. Material growth processes based on molecular beam, and atomic layer epitaxy have been exploited to a great extent in this context, in the past, to prepare superlattices for not only controlling the resultant energy gaps but also arrive at heterostructures supporting 2D-Electron/Hole Gas sheets resulting in very high carrier mobilities in the absence of impurity scattering as discussed already in detail in earlier publication. ${ }^{1}$

With subsequent introduction of nanoscience and technology, the concept of BGE became still better realizable by controlling the size and shape of the nanoparticulate materials (NPMs). ${ }^{2}$ Extension to the idea of preparing comprehensive hierarchical molecular complexes involving further combinations of strong and weak interactions due to conjugations provided better options for preparing nanomaterials possessing smart and intelligent features, which not only employed BGE of the materials species of not only inorganic, and organic (polymeric) origin but also of the biomolecular species to mimic some sort of intelligent behavior of living organisms as discussed in detail in numerous publications. ${ }^{3-6}$
With further advent of 2D layered materials like graphene and graphene like mono/multiple layers, numerous possibilities are foreseen to implement BGE strategy even without bothering for the previously mentioned restrictions of lattice matching (e.g. observed in the heterostructures of (CSM) at the interfaces of two different monolayers resulting in another new class of van der Waal's epitaxial heterostructures in lateral and vertical configurations for improving their electronic/optoelectronic properties. Some of the recent developments discussed here in this review highlight the importance of this growing field of novel 2D-materials from BSE angle. ${ }^{7}$

\section{BGE of nanoparticulate materials}

Semiconducting nanocrystals (NCs) introduce tunable optical transition properties arising out of additional discrete electron states (DES) resulting from quantum confinement. Consequently they are applicable in solar photovoltaic energy conversion, optoelectronic devices, molecular and cellular imaging, and ultrasensitive detection. The light emissions from such NCs vary from UV to visible, to nearinfrared, and mid-infrared spectral ranges. These NCs demonstrate additional properties like carrier multiplication, single-particle blinking, and spectral diffusion and hence used in developing complex superlattices and multimodal agents for molecular imaging and targeted therapy. ${ }^{8}$

The syntheses of these NCs offer numerous types of doped and strain-tuned QDs. Techniques based on single-particle fluorescence, extinction, and tunneling/ultrafast spectroscopies are helping in understanding their characteristic features. For instance, $\mathrm{ZnO}$ having wide band gap with immunity to oxidative degradation is a very useful shell material for NCs capping in core-shell type QDs. Similarly, IV-VI semiconductors having positive deformation potentials and mercury-based II-VI materials allowing for continuous tuning of their band gaps through spontaneous cation exchange reactions are known to play important roles in their future applications. For instance, in photovoltaic applications - the phenomenon like multi-exciton generation causing efficient charge carrier separation; and minimizing the steric hindrance in bio conjugated NCs in biomedical applications. Employing nonspecific protein adsorption for developing photo 
switchable NCs for multicolor optical microscopy in exploring the toxic properties these NCs are some typical examples already known and reported. ${ }^{9,10}$

\section{D-materials for BGE}

A number of options were explored in the recent past after the discovery of graphene in developing engineered nanomaterials by design using 2-D-mono/few-layers of graphene and other materials. Instead of realizing purely uniform homogeneous phases, even patchy compositions of different constituents were formed at isolated locations as identified in structural analyses. The examples cited here show very clearly that once optimal route of synthesis is identified; the utility of the resultant band gap designed nanomaterials will start touching an altogether newer horizon of their applications in near future. ${ }^{11}$

\section{Graphene, h-BN and h-BNC}

Synthesizing large size mono/few layers of graphene, h-BN, and $\mathrm{h}$-BNC in recent experiments have offered good examples cited in this context as described below before highlighting the challenges faced during their hybrid phase syntheses.

Monolayer of graphene is a planar sheet of $\mathrm{sp}^{2}$-bonded carbon atoms hexagonally arranged in a lattice possessing distinct features that are not there in $0 \mathrm{D}, 1 \mathrm{D}$, or $3 \mathrm{D}$ forms of carbon. A variety of physical phenomena were observed including anomalous quantum Hall effect reflecting the presence of mass-less electrons, and possessing high electron mobility at room temperature made it known as a zero gap semiconductor with enhanced electronic properties. Unusual thermal and mechanical properties of graphene have been used in electromechanical resonators, stretchable and elastic matrices for flexible electronic circuitry, ultracapacitors, stable field emitters, and as fillers for electrically conducting flexible nanocomposites. ${ }^{12-18}$

Besides graphene, 2D atomically thin sheets of other materials involve nitrides, sulfides, selenides, tellurides, and oxides. Theoretical calculations predict exceptional properties possible in most of the 2D-materials by reducing them into a monolayer, such as in hexagonal boron nitride (h-BN). Out of several possible phases, h-BN is the only member possessing a layered structure, with the $\mathrm{B}$ and $\mathrm{N}$ atoms arranged in an $\mathrm{sp}^{2}$-honeycomb lattice, quite similar to that of $\mathrm{C}$ atoms in graphene. Structurally identical features of h-BN, graphene, and other 2D-monolayers make them appropriate for fabricating graphene-based electronics and optoelectronics devices. Out of various phases, graphene and h-BN attract much attention as they have demonstrated excellent hybrid phases by mixing them together. Electronically different phases when mixed offer interesting device applications. A controlled mixing of these two lattices in lateral and vertical stacking produces a series of superlattices. Theoretical studies of BNC nanostructures show them usable in nano-electronics, optical devices, field emission, catalysis, lubrication, and gas storage. ${ }^{11,12,19-22}$

The exploration of 2D-BNC atomic layers beyond graphene and $\mathrm{h}$-BN still needs to address to many challenges including preparation of atomic layer phases with precisely controlled compositions, determining the locations of various atoms using scanning tunneling microscopy/spectroscopy (STM/STS), and correlating the measured electronic and magnetic properties to the structural and theoretical predictions. ${ }^{11}$

\section{Other hybrid 2D-monolayers}

Several methods have been attempted in preparing layered phases of $\mathrm{BNC}, \mathrm{BNC}_{2}, \mathrm{BC}_{3}$ by involving synthesis of graphene and h-BN over metal substrates in CVD and other techniques besides codoping of $\mathrm{B}$ and $\mathrm{N}$ into nano carbons. For example, instead of doping graphene with $\mathrm{B}$ and $\mathrm{N}$, it is conversely possible C-doping in h-BN. However, during graphene or h-BN doping, it is essential to realize uniform distribution of the dopants to have the engineered phases. The unusual properties of graphene have triggered interest in doping the hexagonal lattice of graphene with boron $(\mathrm{B})$ and nitrogen $(\mathrm{N})$ to obtain layered BNC-structures. Individual atomic layers containing B, $\mathrm{C}$, and $\mathrm{N}$ of various compositions conform to several stable phases in the three-component phase diagram of B-C-N. Additionally, stacking layers built from $\mathrm{C}$ and $\mathrm{BN}$ also allows for engineering the new vander-Waals materials with novel properties. ${ }^{11}$

BGE strategy has been explored in 2D-materials to reduce the large band gap of h-BN monolayer (i.e. $\sim 6 \mathrm{eV}$ ) by topologically modifying its hexagonal lattice by creating grain boundaries (GBs) in its basal plane. These monolayers have 3-fold symmetry with alternative $\mathrm{B}$ and $\mathrm{N}$ atoms in the pristine form. The edges and GBs in such a lattice show characteristic optical/electronic properties. Lesser investigated GBs in h-BN compared to those in graphene and $\mathrm{MoS}_{2}$ are identified as an array of dislocations and non-hexagonal boron and nitrogen rings, which ultimately disturb the lattice symmetry causing reductions in the band gaps in some orientations. DFT calculations involving additional states created along the boundary estimate the BG reductions up to $\sim 38 \%$. In the alternative option, it was substituting $\mathrm{C}$ atoms to form hybrid lattice of h-BNC in place of h-BN. A general chemical method reported in this context to prepare different h-BN derivatives confirmed that the chemical structure could very well be modified with or without a graphene precursor to tune the BG up to $\sim 2 \mathrm{eV}^{23-25}$

Besides, the processes like atomic doping, alloying and hybridization were also attempted in past to alter electronic structures of h-BN monolayers by seamless inter-weaving of graphene and h-BN into one piece. Earlier studies have confirmed that B, C and N could be atomically mixed together to form various semiconducting 2D-monolayer structures with varying stoichiometry. The electronic and magnetic properties of $\mathrm{BN}$ monolayer are theoretically possible to modulate by adding small domains of graphene in h-BN, without disturbing its planarity. In recent experiments, co-deposition of graphene and h-BN was attempted to form hybridized h-BNC besides synthesizing atomic layers of h-BNC by substituting $\mathrm{C}$ atoms in graphene layer with $\mathrm{B}$ and $\mathrm{N}$ atoms. The significance of the chemical and electrical tunability achieved in these experimental methods motivated using PECVD to synthesize several other members of h-BN derivatives. ${ }^{23,26-29}$

For the introduction of h-BN domain in a pristine graphene monolayer, hydrogen is used to etch away $\mathrm{C}$ atoms generating vacancies that are filled by $\mathrm{B}$ and $\mathrm{N}$ atoms ultimately leading to the growth of h-BN domains. Continuing this process over a sufficiently longer duration, the graphene monolayer is completely converted into h-BN film. This kind of gradual change taking place in the graphene lattice has been confirmed, for instance, by starting with a pristine graphene film with no D peak (i.e. indicating absence of a detectable defect), the gradual conversion gives rise to the appearance of D-peak that increases in the early $40 \mathrm{~min}$ and declines afterwards. These 
characteristics are in accordance with the increasing levels of defects or functionalities in graphene. It is quite likely that hybrid structure are constituted with alloys or mixed domains of h-BN, graphene and h-BNC. Especially, when the conversion exceeds $15 \mathrm{~min}$, the D peak of graphene starts to slowly blue shift from $1354 \mathrm{~cm}^{-1}$ to the h-BN's E2g peak at $1368 \mathrm{~cm}^{-1} \cdot 23,26,30,31$

BGE of bulk compound semiconductors (e.g. binary, ternary, quaternary and so on) using alloying of constituent crystalline compounds with matching lattice constants has already been explored in past facilitating their applications in numerous electronic/ optoelectronic devices to a large extent. ${ }^{1}$ Drawing a parallel from there motivated to try similar attempts in atomically thin $2 \mathrm{D}$ semiconductor monolayers to grow materials with tunable band gaps for their applications in several functional devices. ${ }^{1,32-41}$

In this context, a number of monolayers (2D) with different energy gaps when alloyed were found to produce hybrid 2D materials possessing stoichiometry dependent BGs lying somewhere between the minimum and maximum limits of the band gaps of the constituent compounds used. Such hybrid materials are finding applications in nanoelectronics and nano photonics, where it is rather beneficial to have semiconducting nanostructures with continuously tunable band gaps. Recent advances in $0 \mathrm{D}$ and $1 \mathrm{D}$ ternary semiconductor structures have shown that their band gaps and light emissions could also be tuned gradually by changing their constituent stoichiometries, and based on these composition modulated nanostructures, multiwavelength lasers, wavelength converters, optical diodes, and nano photodetectors have been realized experimentally. ${ }^{42-52}$ For their applications in integrated devices and systems, direct growth of these band gap engineered 2D monolayer materials are proving to be very useful as discussed in a recent review covering the recent progress on the BGE of atomically thin 2D semiconductor alloys. Some of the typical examples are included in the followings to highlight their future applications. ${ }^{32}$

\section{Other hybrid 2D-materials}

Thermodynamically stable alloys of sulphides, and selenides of Mo and $\mathrm{W}$ were, for instance, studied in great detail by varying their stoichiometries. For instance, $\mathrm{MoS}_{2(1-x)} \mathrm{Se}_{2 \mathrm{x}}$ was studied for fixing its band gap between 1.557 and $1.856 \mathrm{eV}$ (i.e. corresponding to the direct band gap of $\mathrm{MoSe}_{2}$ and $\mathrm{MoS}_{2}$, respectively). ${ }^{53,54}$ Atomically thin nanosheets of 2D-MoS ${ }_{2(1-x)} \mathrm{Se}_{2 \mathrm{x}}$ were also examined for lithium adsorption and diffusion in hexagonal monolayers for different values of $x$ as: $0.00,0.33,0.50,0.66$, and 1.00 . Band gap engineered nanosheets were successfully produced using precursors from mechanically exfoliated $\mathrm{MoS}_{2(1-\mathrm{x})} \mathrm{Se}_{2 \mathrm{x}}$ bulk alloy showing triangular nanosheets (SEM) with the edge lengths of 30-80micron. Lateral compositiongraded $2 \mathrm{D}-\mathrm{MoS}_{2(1-\mathrm{x})} \mathrm{Se}_{2 \mathrm{x}}$ nano layers were also synthesized employing a simple moving source thermal evaporation method of an improved CVD. ${ }^{55-68}$ Subsequently, 2D-WS ${ }_{2 x} \mathrm{Se}_{2(1-x)}$ alloys were prepared from $\mathrm{WO}_{3}$ (monoclinic crystal) to $\mathrm{WS}_{2(1-x)} \mathrm{Se}_{2 \mathrm{x}}$ (hexagonal crystal) through simultaneous sulfurization and selenization, in which, the chemical activity of S made it easy to tune the value of $x$ by varying the weight of sulfur powder. ${ }^{69}$ The resulting alloy nanosheets were monolayers with a well-defined triangular shape, as identified by optical contrast and atomic force microscopy besides Raman spectroscopy. ${ }^{69}$

\section{Some recent findings}

Some recent experimental studies conducted by various research groups are described here in brief to highlight the validations of theoretical computations regarding $\mathrm{BGE}$ aspects of 2D-monolayeres. Particularly, strain induced changes in material and device characteristics are very valuable informations regarding this aspect of material by design using 2D-mono/multiple layer materials in association with graphene.

Synthesis of monolayers containing hybridized bonds of elements $\mathrm{B}, \mathrm{N}$ and $\mathrm{C}$ are expected to offer new material properties as discussed earlier. Synthesis and characterization of large-area atomic layers of $h$-BNC material was reported in case of hybridized, randomly distributed domains of $h$ - $\mathrm{BN}$ and $\mathrm{C}$ phases with compositions ranging from pure $\mathrm{BN}$ to pure graphene indicating their distinct structural features and band gap from those of undoped/doped graphene, and $h$-BN, which is found useful for developing band gap-engineered device applications. ${ }^{27}$

In a recent study of 2-D B $\mathrm{B}_{x / 2} \mathrm{~N}_{x / 2} \mathrm{C}_{1-x}(0<x<1)$ compounds with four stoichiometric compositions namely: $\mathrm{B}_{x / 2} \mathrm{~N}_{x / 2} \mathrm{C}_{1-x}(x=2 / 3,1 / 2,2 / 5$ and $1 / 3)$ was reported using a global optimization method (CALYPSO) and DFT revealing that all monolayer $\mathrm{B}_{x / 2} \mathrm{~N}_{x / 2} \mathrm{C}_{1-x}$ stoichiometries adopt a planar honeycomb character and are dynamically stable. These calculations show that most of the $\mathrm{B}_{x / 2} \mathrm{~N}_{x / 2} \mathrm{C}_{1-x}$ phases possess direct band gaps within the optical range; thereby they can potentially be used in high-efficiency conversion of solar energy in photovoltaic modules. The band gaps of $\mathrm{B}_{x / 2} \mathrm{~N}_{x / 2} \mathrm{C}_{1-x}$ can be widely tuned within the optical range by changing the concentration of carbon, thus allowing the fast development of BGE in optoelectronics. $^{70}$

In one method of BG-tuning of monolayer h-BN $(\sim 6 \mathrm{eV})$ involves topologically reforming the hexagonal structure incorporating grain boundaries (GBs) in the basal plane. Alternately, introducing foreign atoms like $\mathrm{C}$ forges hybrid structures like hetero-junctions or semiconducting h-BNC materials. A general chemical method of synthesizing these different versions of h-BN derivatives was showcased for manipulating the chemical structure either with or without a graphene precursor, resulting in $\mathrm{BG}$ down to $\sim 2 \mathrm{eV}^{71}$

The key to have exotic features from van der Waals 2D-semiconductors for device applications lies in precisely modifying their BGs. The strategy of universally tuning the band gap in the family of bulk $2 H$ transition metal dichalcogenides (TMDs) was reported recently by in-situ surface doping of $\mathrm{Rb}$ atoms. Experimental observations confirmed a variation in band gap in the range of 0.8 $2.0 \mathrm{eV}$, which covers a wide spectral range from visible to near infrared, with a tendency from indirect to direct BGs. These results confirmed surface Stark effect as a universal mechanism of band-gap engineering on the basis of the strong $2 \mathrm{D}$ nature of van der Waals semiconductors. $^{72}$

$\mathrm{MoTe}_{2}$ is an exfoliable TMD crystallizing in three symmetries: semiconducting trigonal-prismatic $2 \mathrm{H}$ - or $\alpha$-phase; semi metallic and monoclinic 1T'- or $\beta$-phase; and semi metallic orthorhombic $\gamma$-structure. Out of these the $2 \mathrm{H}$-phase has a band gap of $\sim 1 \mathrm{eV}$ making ideal for flexible and transparent optoelectronic applications. The $\gamma$-phase possesses a topologically protected non-dissipative transport channels along with possibility of locally inducing phasetransformations in TMDs, through chemical doping, local heating, or electric field to achieve ohmic contacts. It is also feasible to induce functionalities like electronic phase-change memory elements. Combining these features, it is possible to use this material for producing high performance, low dissipation optoelectronic devices in near future. Phase engineering of $\mathrm{MoTe}_{2}$ by W-substitution was revealed in the phase-diagram of the $\mathrm{Mo}_{1-\mathrm{x}} \mathrm{W}_{\mathrm{x}} \mathrm{Te}_{2}$ solid solution, which 
displays a semiconducting to semi metallic transition as a function of $x$. A small critical W concentration $x_{c} \sim 8 \%$ was observed to stabilize the $\gamma$-phase at room temperature suggesting that crystals with $x$ close to $x_{c}$ might be particularly susceptible to phase transformations induced by an externally applied electric field. ${ }^{73}$

While examining 2d-monolayers, tailoring graphene/boron nitride heterostructures, which can theoretically retain the character of a single-atom thick sheet, withstand large physical strains, are easily functionalized, and have entirely different optical and mechanical properties compared to graphene providing the foundation for entirely new research fields. Knowing from theoretical predictions that carbon, boron, and nitrogen could co-exist as atomic sheets in a layered structure, a facile method of integrating boron nitride $(\mathrm{hBN})$ and graphene oxide (GO) via chemical exfoliation was reported in form of BCON after examining its stability at different $\mathrm{pH}$ conditions indicating that a stable and a uniform solution is achievable at $\mathrm{pH}$ in the range of 4 to 8. An in-situ XPS was employed in detecting chemical changes while exposing it to ionization radiation specially focusing on the $\mathrm{C} / \mathrm{O}$ ratio. It was observed that even with a very low energy source, this material is highly sensitive to ionizing radiation, such as neutron, alpha and beta particles. ${ }^{74}$

A continuous and reversible tuning of the optical band gap of suspended $\mathrm{MoS}_{2}$ monolayer was reported by as much as $500 \mathrm{meV}$ by applying very large biaxial strains. Impermeability to gas of CVD grown crystals was used to apply a pressure difference across suspended membranes to induce biaxial strains for determining the effect of strain on the energy and intensity of the peaks in the photoluminescence (PL) spectrum and find a linear tuning rate of the optical band gap of $99 \mathrm{meV} / \%$. These results show that as large as $5.6 \%$ strain could be applied across micron-sized areas showing strain tuning of higher-level optical transitions. ${ }^{75}$

Continuous tuning of the electronic structure of atomically thin $\mathrm{MoS}_{2}$ on flexible substrates was reported by applying a uniaxial tensile strain with a redshift @ $~ 70 \mathrm{meV} / \%$ applied strain for direct gap transitions, and @ 1.6 times larger for indirect gap transitions, as determined by absorption and photoluminescence spectroscopy. These results agree with first principles calculations demonstrating the utility of 2D- monolayers in flexible and optoelectronics. ${ }^{76}$

Substantial strain induced band gap tunability was demonstrated in TMDs based FETs as per theoretical calculations and optical experiments. Devices fabricated on flexible substrates held on cantilever type sample holder were used to apply uniaxial tensile strain. The analysis of FET's transfer characteristics clearly proved a band gap reduction of $100 \mathrm{meV}$ in $\mathrm{WSe}_{2}$ under $1.35 \%$ uniaxial tensile strain at room temperature in which the band gap change is only related to a change of the conduction band edge of WSe, resulting in a decrease in the Schottky barrier (SB) for electrons without any change for hole injection into the valence band. These experimental results, when compared with DFT calculations under strain, showed excellent agreement between theoretical predictions and the experimental data presented here. ${ }^{77}$

\section{Conclusion}

Developing appropriate synthesis techniques with precise control of monolayers of 2-D layered materials by combining binary and ternary phases of $\mathrm{C}, \mathrm{B}$, and $\mathrm{N}$ in hybrid form is theoretically predicted to tune the BGs and hence their electronic, optoelectronic and other structural properties as highlighted in this work. This could possibly be better explored than tuning the BGs using nanoparticulate materials building blocks alone as discussed in earlier publications. A combination of nanoparticulate materials and band gap engineered 2D-monolayers forming hierarchical molecular complexes would be a better option as it appears from the observations made so far.

\section{Acknowledgements}

None.

\section{Conflict of interest}

The author declares no conflict of interest.

\section{References}

1. S Ahmad. Microwave and Millimeter Wave Semiconductor Materials Technology. IETE monograph; Vol VI; New Delhi, India: Tata McGraw-Hill Publishing Co. Ltd.; 1998. p. 1-180.

2. Talapin DV, Lee JS, Kovalenko MV, et al. Prospects of colloidal nanocrystals for electronic and optoelectronic applications. Chem Rev. 2010;110(1):389-458.

3. Ahmad S. Materials by design-Prospects and challenges. Indian J Engineering \& Materials Sciences. 2005;12(4):299-316.

4. Ahmad S. Device applications of band-structure-engineered nanomaterials current status and future trend-review. Int J Nanoelectronics and Materials. 2015;8(2):129-202.

5. Ahmad S. Engineered nanomaterials for drug and gene delivery-a review. J Nanopharmaceutics and Drug Delivery. 2016;3(1):1-50.

6. Ahmad S. Band-structure-engineered materials synthesis. Int J Material Science. 2016;6(1):1-34.

7. Das S, Robinson JA, Dubey M, et al. Beyond graphene: progress in novel two-dimensional materials and van der waals solids. Annual Rev Mater Res. 2015;45:1-27.

8. Gomez DE, Califano M, Mulvaney P. Optical properties of single semiconductor nanocrystals. Phys Chem Chem Phys. 2006;8(43):49895011 .

9. Kukura P, Celebrano M, Renn A, et al. Imaging a single quantum dot when it is dark. Nano Lett. 2009;9(3):926-929.

10. Smith AM, Duan HW, Mohs AM, et al. Bioconjugated quantum dots for in vivo molecular and cellular imaging. Adv Drug Del Rev. 2008;60(11):1226-1240.

11. Song L, Liu Z, Reddy ALM, et al. Binary and ternary atomic layers built from carbon, boron, and nitrogen. Adv Mater. 2012;24(36):4878-4895.

12. Dreyer DR, Ruoff RS, Bielawski CW. From conception to realization: an historial account of graphene and some perspectives for its future. Angew Chem Int Ed. 2010;49(49):9336-9344.

13. Castro Neto AH, Guinea F, Peres NMR, et al. The electronic properties of graphene. Rev Mod Phys. 2009;81(1):109

14. Das Sarma S, Adam S, Hwang EH, et al. Electronic transport in two-dimensional graphene. Rev Mod Phys. 2011;83(2):407.

15. Novoselov KS, Geim AK, Morozov SV, et al. Electric field effect in atomically thin carbon films. Science. 2004;306(5696):666-669.

16. Geim AK, Novoselov KS. The rise of graphene. Nat Mater. 2007;6(3):183-191. 
17. Novoselov KS, Geim AK, Morozov SV, et al. Two-dimensional gas of massless Dirac fermions in graphene. Nature. 2005;438(7065):197-200.

18. Lee C, Li Q, Kalb W, et al. Frictional characteristics of atomically thin sheets. J Hone Science. 2010;328(5974):76-80.

19. Novoselov KS, Jiang D, Schedin F, et al. Two-dimensional atomic crystals. Proc Natl Acad Sci U S A. 2005;102(30):10451-10453.

20. Coleman JN, Lotya M, O’Neill A, et al. Two-dimensional nanosheets produced by liquid exfoliation of layered materials. Science. 2011;331(6017):568-571

21. Najmaei S, Liu Z, Ajayan PM. J Lou Appl Phys ett. 2012;100:013106.

22. Corso M, Auwärter W, Muntwiler M, et al. Boron nitride nanomesh. Science. 2004;303(5655):217-220.

23. Ba K, Jiang W, Cheng J, et al. Chemical and band gap engineering in monolayer hexagonal boron nitride. Sci Rep. 2017;7:45584.

24. Liu Y, Zou X, Yakobson BI. Dislocations and grain boundaries in two-dimensional boron nitride. ACS Nano. 2012;6(8):7053-7058.

25. Li Q, Zou X, Liu M, et al. Grain boundary structures and electronic properties of hexagonal boron nitride on $\mathrm{Cu}(111)$. Nano Lett. 2015;15(9):5804-5810.

26. Gong Y, Shi G, Zhang Z, et al. Nature Commun. 2014;5:3193.

27. Ci L, Song L, Jin C, et al. Atomic layers of hybridized boron nitride and graphene domains. Nature Mater. 2010;9(5):430-435.

28. Kan M, Zhou J, Wang Q, et al. Phys Rev B. 2011;84:205412.

29. Sutter P, Cortes R, Lahiri J, et al. Interface formation in monolayer graphene-boron nitride heterostructures. Nano Lett. 2012;12(9):48694874.

30. Ferrari AC, Meyer JC, Scardaci V, et al. Raman spectrum of graphene and graphene layers. Phys Rev Lett. 2006;97(18):187401.

31. Englert JM, Vecera P, Knirsch KC, et al. Scanning-Raman-microscopy for the statistical analysis of covalently functionalized graphene. ACS Nano. 2013;7(6):5472-5482.

32. Ge $\mathrm{CH}$, Li HL, Zhu XL, et al. Band gap engineering of atomically thin two-dimensional semiconductors. Chin Phys B. 2017;26(3):034208.

33. Gong Y, Lin J, Wang X, et al. Vertical and in-plane heterostructures from WS2/MoS2 monolayers. Nat Mater. 2014;13(2):1135-1142.

34. Duan X, Wang C, Shaw J, et al. Lateral epitaxial growth of two-dimensional layered semiconductor heterojunctions. Nat Nanotechnol. 2014;9(12):1024-1030

35. Li M, Shi Y, Cheng C, et al. Science. 2015;49:524.

36. Huang C, Wu S, Sanchez A, et al. Lateral heterojunctions within monolayer MoSe2-WSe2 semiconductors. Nat Mater. 2014;13(12):109610101.

37. Zhang XQ, Lin CH, Tseng YW, et al. Synthesis of lateral heterostructures of semiconducting atomic layers. Nano Lett. 2015;15(1):410-415.

38. Gong Y, Lei S, Ye G, et al. Two-step growth of two-dimensional WSe2/ MoSe2 heterostructures. Nano Lett. 2015;15(9):6135-6141.

39. Xie LM. Two-dimensional transition metal dichalcogenide alloys: preparation, characterization and applications. Nanoscale. 2015;7(44):18392-18401.

40. Shi Y, Li H, Li LJ. Recent advances in controlled synthesis of two-dimensional transition metal dichalcogenides via vapour deposition techniques. Chem Soc Rev. 2015;44(9):2744-2756.
41. Kutana A, Penev ES, Yakobson BI. Nanoscale. 2014;6:5820.

42. Pan A, Yang H, Yu R, et al. Nanotechnology. 2006;17:1083.

43. Pan AL, Yao L, Qin Y, et al. Si-CdSSe core/shell nanowires with continuously tunable light emission. Nano Lett. 2008;8(10):3413-3417.

44. Pan A, Liu R, Sun M, et al. Quaternary alloy semiconductor nanobelts with band gap spanning the entire visible spectrum. J Am Chem Soc. 2009;131(27):9502-9503.

45. Pan A, Liu R, Sun M, et al. Spatial composition grading of quaternary $\mathrm{ZnCdSSe}$ alloy nanowires with tunable light emission between 350 and $710 \mathrm{~nm}$ on a Single Substrate. ACS Nano. 2010;4(2):671-680.

46. Gu F, Yang Z, Yu H, et al. Spatial band gap engineering along single alloy nanowires. J Am Chem Soc. 2011;133(7):2037-2039.

47. Yang $\mathrm{Z}, \mathrm{Xu} \mathrm{J}$, Wang $\mathrm{P}$, et al. On-nanowire spatial band gap design for white light emission. Nano Lett. 2011;11(11):5085-5089.

48. Zhuang X, Ning CJ, Pan. Composition and band gap-graded semiconductor alloy nanowires. Adv Mater. 2012;24(1):13-33.

49. Wang Y, Xu J, Ren P, et al. Phys Chem Chem Phys. 2013;15:2912.

50. Guo $\mathrm{P}, \mathrm{Hu} \mathrm{W}$, Zhang Q, et al. Semiconductor alloy nanoribbon lateral heterostructures for high-performance photodetectors. Adv Mater. 2014;26(18):2844-2849.

51. Ma L, Hu W, Zhang Q, et al. Room-temperature near-infrared photodetectors based on single heterojunction nanowires. Nano Lett. 2014;14(2):694-698.

52. Guo P, Xu J, Gong K, et al. On-nanowire axial heterojunction design for high-performance photodetectors. ACS Nano. 2016;10(9):8474-8481.

53. Komsa HP, Krasheninnikov AV. Two-dimensional transition metal dichalcogenide alloys: stability and electronic properties. J Phys Chem Lett. 2012;3(23):3652-3656.

54. Ersan F, Gokoglu G, Akturk E. Adsorption and diffusion of lithium on monolayer transition metal dichalcogenides $\left(\mathrm{MoS}_{2(1-\mathrm{x})} \mathrm{Se}_{2 \mathrm{x}}\right)$ alloys. $J$ Phys Chem C. 2015;119(51):28648-28652.

55. Kou L, Frauenheim T, Chen C. Nanoscale multilayer transition-metal dichalcogenide heterostructures: band gap modulation by interfacial strain and spontaneous polarization. J Phys Chem Lett. 2013;4(10):17301736.

56. Tan CL, Zhao W, Chaturvedi A, et al. Preparation of single-layer $\mathrm{MoS}_{2 \mathrm{x}}$ $\mathrm{Se}_{2(1-x)}$ and $\mathrm{Mo}_{\mathrm{x}} \mathrm{S}_{2}$ nanosheets with high-concentration metallic 1T phase. Small. 2016;12(14):1866-1874.

57. Feng Q, Zhu Y, Hong J, et al. Growth of large-area $2 \mathrm{D} \operatorname{MoS}_{2(1-x)} \operatorname{Se}_{2 x}$ semiconductor alloys. Adv Mater. 2014;26(17):2648-2653.

58. Li H, Duan X, Wu X, et al. Growth of Alloy $\operatorname{MoS}_{2 \mathrm{x}} \mathrm{Se}_{2(1-\mathrm{x})}$ Nanosheets with Fully tunable chemical compositions and optical properties. $J$ Am Chem Soc. 2014;136(10):3756-3759.

59. Gong Y, Liu Z, Lupini AR, et al. Band gap engineering and layer-by-layer mapping of selenium-doped molybdenum disulfide. Nano Lett. 2014;14(2):442-449.

60. Ma Q, Isarraraz M, Wang CS, et al. Postgrowth tuning of the band gap of single-layer molybdenum disulfide films by sulfur/selenium exchange. ACS Nano. 2014;8(5):4672-4677.

61. Li H, Zhang Q, Duan X, et al. Lateral growth of composition graded atomic layer $\mathrm{MoS}_{2(1-\mathrm{x})} \mathrm{Se}_{2 \mathrm{x}}$ nanosheets. $J$ Am Chem Soc. 2015;137(16):5284-5287. 
62. Chen X, Wang Z, Qiu Y, et al. J Mater Chem A. 2016;4:18060.

63. Zhang W, Li X, Jiang T, et al. CVD synthesis of $\operatorname{Mo}((1-x)) W_{(x)} S_{2}$ and $\mathrm{MoS}_{(2(1-\mathrm{x}))} \mathrm{Se}_{(2 \mathrm{x})}$ alloy monolayers aimed at tuning the band gap of molybdenum disulfide. Nanoscale. 2015;7(32):13554-13560.

64. Feng Q, Mao N, Wu J, et al. Growth of $\operatorname{MoS}_{2(1-x)} \operatorname{Se}_{2 \mathrm{x}}(\mathrm{x}=0.41-1.00)$ monolayer alloys with controlled morphology by physical vapor deposition. ACS Nano. 2015;9(7):7450-7455.

65. Xia B, An L, Gao D, et al. Cryst Eng Comm. 2015;17:6420.

66. Mann J, Ma Q, Odenthal PM, et al. 2-dimensional transition metal dichalcogenides with tunable direct band gaps: $\mathrm{MoS}_{2(1-\mathrm{x})} \mathrm{Se}_{2 \mathrm{x}}$ monolayers. Adv Mater. 2014;26(9):1399-1404.

67. Fu Q, Yang L, Wang W, et al. Synthesis and enhanced electrochemical catalytic performance of monolayer $\mathrm{WS}_{2(1-\mathrm{x})} \mathrm{Se}_{2 \mathrm{x}}$ with a tunable band gap. Adv Mater. 2015;27(32):4732-4738.

68. Huang J, Wang W, Fu Q, et al. Stable electrical performance observed in large-scale monolayer $\mathrm{WSe}_{2(1-\mathrm{x})} \mathrm{S}_{2 \mathrm{x}}$ with tunable band gap. Nanotechnology. 2016;27(13):13LT01.

69. Duan X, Wang C, Fan Z, et al. Synthesis of $\mathrm{WS}_{2 \mathrm{x}} \mathrm{Se}_{2-2 \mathrm{x}}$ Alloy nanosheets with composition-tunable electronic properties. Nano Lett. 2015;16(1):264-269.
70. Zhang M, Gao G, Kutana A, et al. Nanoscale. 2015;7:12023-12029.

71. Ba K, Jiang W, Cheng J et al. Sci Rep. 2017;7:45584.

72. Kang M, Kim B, Ryu SH, et al. Universal mechanism of bandgap engineering in transition-metal dichalcogenides. Nano Lett. 2017;17(3):1610-1615.

73. Rhodes D, Chenet DA, Janicek BE, et al. Engineering the structural and electronic phases of $\mathrm{MoTe}_{2}$ through W substitution. Nano Lett. 2017;17(3):1616-1622.

74. Bhimanapati GR, Wetherington M, Mahabir S, et al. Synthesis and radiation response of BCON: a graphene oxide and hexagonal boron nitride hybrid. 2D Materials. 2016;3(2):025028.

75. Lloyd D, Liu X, Christopher JW, et al. Band gap engineering with ultralarge biaxial strains in suspended monolayer $\mathrm{MoS}_{2}$. Nano Lett. 2016;16(9):5836-5841.

76. He K, Poole C, Mak KF, et al. Experimental demonstration of continuous electronic structure tuning via strain in atomically thin $\mathrm{MoS}_{2}$. Nano Lett. 2013;13(6):2931-2936.

77. Shen T, Penumatcha AV, Appenzeller J. Strain engineering for transition metal dichalcogenides based field effect transistors. ACS Nano. 2016;10(4):4712-4718 